\title{
A SEMINORM WITH SQUARE PROPERTY ON A BANACH ALGEBRA IS SUBMULTIPLICATIVE
}

\author{
S. J. BHATT
}

(Communicated by Palle E. T. Jorgensen)

\begin{abstract}
The result stated in the title is proved in a Banach algebra and is used to discuss (i) commutativity criteria in normed algebras, (ii) uniqueness of the uniform norm in uniform Banach algebras, and (iii) existence of continuous multiplicative linear functionals on topological algebras together with a simple reduction of the Michael problem in Fréchet algebras. Submultiplicativity does not imply subadditivity in the presence of the square property.
\end{abstract}

Theorem. Let $p$ be a seminorm with the square property on a Banach algebra $A$. Then the following hold.

(1) $p$ is continuous on $A$.

(2) $N_{p}=\{x \in A \mid p(x)=0\}$ is a closed two-sided ideal in $A$.

(3) $p(x y) \leq p(x) p(y)$ for all $x, y$ in $A$.

(4) The quotient algebra $A / N_{p}$ is commutative.

The above theorem answers, in the particular case of Banach algebras, a problem posed in [3]. A seminorm on $A$ is a function $p: A \rightarrow[0, \infty)$ satisfying (i) $p(x+y) \leq p(x)+p(y)$ for all $x, y$ and (ii) $p(\lambda x)=|\lambda| p(x)$ for all $x$, for all scalars $\lambda$. It satisfies the square property [3] if (iii) $p\left(x^{2}\right)=p(x)^{2}$ for all $x$. It is submultiplicative if (iv) $p(x y) \leq p(x) p(y)$ for all $x, y$. We shall use [3, Theorem 1], viz. a seminorm with the square property on a commutative linear associative algebra is automatically submultiplicative.

Proof of the theorem. For $x, y$ in $A,(x+y)^{2}=x^{2}+y^{2}+x y+y x$ implies that

$$
\begin{aligned}
p(x y+y x) & \leq p\left((x+y)^{2}\right)+p\left(x^{2}\right)+p\left(y^{2}\right) \\
& =(p(x+y))^{2}+p(x)^{2}+p(y)^{2} \\
& \leq(p(x)+p(y))^{2}+p(x)^{2}+p(y)^{2} \\
& =2\left[p(x)^{2}+p(y)^{2}+p(x) p(y)\right] .
\end{aligned}
$$

Let $x \in N_{p}, y \in A$. Then $p(x y+y x) \leq 2 p(y)^{2}$. But $N_{p}$ being a subspace of

Received by the editors June 10, 1991.

1991 Mathematics Subject Classification. Primary 46H05, 46J05.

Key words and phrases. Seminorm with the square property on a Banach algebra, submultiplicative seminorm, Michael problem in Fréchet algebras. 
$A, n x \in N_{p}$ for all $n \in \mathbb{N}$, with the result

$$
p(x y+y x) \leq \frac{2}{n} p(y)^{2} \rightarrow 0 \quad \text { as } n \rightarrow \infty .
$$

Thus $x \in N_{p}, y \in A$ implies $x y+y x \in N_{p}$.

Again for arbitrary $x, y$ in $A$, consider the identity $(x y-y x)^{2}+(x y+y x)^{2}=$ $2[x(y x y)+(y x y) x]$

Then

$$
\begin{aligned}
p(x y-y x)^{2} & =p\left((x y-y x)^{2}\right) \\
& \leq p\left\{(x y-y x)^{2}+(x y+y x)^{2}\right\}+p\left((x y+y x)^{2}\right) \\
& =2 p[x(y x y)+(y x y) x]+p(x y+y x)^{2} .
\end{aligned}
$$

By the above, if $x \in N_{p}, y \in A$, then $x(y x y)+(y x y) x \in N_{p}, x y+y x \in N_{p}$; hence $x y-y x \in N_{p}$ by this inequality. Thus $x y \in N_{p}, y x \in N_{p}$, and $N_{p}$ is an ideal.

Now $\left|x_{p}\right|=p(x) \quad\left(x_{p}=x+N_{p}\right)$ defines a norm on the quotient algebra $A_{p}=$ $A / N_{p}$. Let $x \in A, u=x_{p}$, and let $M$ be a maximal commutative subalgebra of $A / N_{p}$ containing $u$. Then by [3, Theorem 1], $|\cdot|$ is submultiplicative on $M$ and $(M,|\cdot|)$ is a normed algebra with $|\cdot|$ satisfying the square property. Let $\operatorname{sp}_{K}(\cdot)$ and $\mathrm{r}_{K}(\cdot)$ denote, respectively, the spectrum and the spectral radius in an algebra $K$. Then $|u|=\lim \sup _{n}\left|u^{2^{n}}\right|^{1 / 2^{n}}=\lim \sup _{n}\left|u^{n}\right|^{1 / n} \leq r_{M}(u)$ [4, Theorem 5.7, p. 22]. By the maximality of $M$, [4, Theorem 16.4, p. 76] gives $\mathrm{sp}_{M}(u)=\mathrm{sp}_{A_{p}}(u)$, hence $r_{M}(u)=r_{A_{p}}(u)$. Also, in view of the homomorphism $\pi: A \rightarrow A_{p}, \pi(z)=z_{p}, \operatorname{sp}_{A_{p}}(u) \subset \operatorname{sp}_{A}(x)$. It follows that $p(x)=|u| \leq r_{A}(x) \leq$ $\|x\|$ for any $x \in A,\|\cdot\|$ denoting the Banach algebra norm on $A$. Thus $p$ is continuous and $N_{p}$ is closed. Also by [2, Lemma 2, p. 46], $p(x y-y x)=0$ for all $x, y$ in $A$, with the result that $A_{p}$ is commutative. Hence, again by [3, Theorem 1], $|\cdot|$ is submultiplicative on $A_{p}$, which implies that $p(x y) \leq$ $p(x) p(y)$ for all $x, y$ in $A$. This completes the proof.

By passing to the completion, the following can be verified.

Corollary. Let $p$ be a continuous seminorm with the square property on a normed algebra $A$. Then $N_{p}$ is a two-sided ideal in $A ; p(x y) \leq p(x) p(y)$ for all $x, y$ in $A$; and $A / N_{p}$ is commutative.

Remarks. (1) The above result is a uniform seminorm analogue of a result of Sebestyen [10] that a seminorm $p$ on any *-algebra $A$ (not necessarily a Banach algebra) satisfying $p\left(x^{*} x\right)=p(x)^{2}$ for all $x$ is submultiplicative and $p\left(x^{*}\right)=p(x)$ holds for all $x$. Does the above theorem hold for any linear associative algebra $A$ ?

(2) The property (iv) does not imply (i) in the presence of (ii) and (iii). Take $A=A(D)$, the disc algebra with pointwise operations consisting of all continuous functions on the closed unit disc $D$ that are holomorphic in the interior of $D . A$ is a Banach ${ }^{*}$-algebra with supnorm

$$
\|f\|_{\infty}=\sup \{|f(z)| \mid z \in D\} \text {, }
$$

and the involution $f \rightarrow f^{*}, f^{*}(z)=\overline{f(\bar{z})}$. The involution is not hermitian [2, p. 114]. Let $s(f)=r\left(f^{*} f\right)^{1 / 2}$. Then $s(\cdot)$ satisfies (ii), (iii), (iv) and the $C^{*}$-property $s\left(f^{*} f\right)=s(f)^{2}$ for all $f$. However, $s(\cdot)$ does not satisfy (i); for 
otherwise, $s(\cdot)$ would become a $C^{*}$-seminorm, with the result, by [4, Lemma 39.2 , p. 214], that $s(\cdot)=m(\cdot)$, the greatest $C^{*}$-seminorm, forcing $A$ to be hermitian [4, Theorem 41.11 , p. 227].

(3) It follows from the above theorem that if $A$ is a Banach algebra in which the numerical radius $\nu(\cdot)$ satisfies (iii), then $A$ is commutative and $r(\cdot)=\nu(\cdot)$.

(4) Let $(A,\|\cdot\|)$ be a normed algebra. Then for all $x, r^{\prime}(x)=\varlimsup \lim \left\|x^{n}\right\|^{1 / n} \leq$ inf $\left\|x^{n}\right\|^{1 / n} \leq\|x\|\left[4\right.$, Proposition 2.8, p. 11] and $r^{\prime}\left(x^{2}\right)=r^{\prime}(x)^{2}$. If $r^{\prime}(\cdot)$ satisfies (i) then $r^{\prime}(\cdot)$ satisfies (iv) and $A / N_{r^{\prime}}$ is commutative. This is a normed algebra version of a part of [2, Theorem 2, p. 48].

(5) Let $A=C(X)$, the Banach algebra with supnorm $\|\cdot\|_{\infty}$ of all continuous functions on a compact Hausdorff space $X$. Let $|\cdot|$ be a norm on $C(X)$ with square property. Then $|\cdot|$ is submultiplicative and $|\cdot| \leq\|\cdot\|_{\infty}$. But by $[8$, Theorem 1.2 .4 , p. 5], $\|\cdot\|_{\infty} \leq|\cdot|$. Thus $\|\cdot\|_{\infty}$ is the only norm with square property on $C(X)$ making it a normed linear space; and by [3], this does not hold for a proper uniformly closed nonselfadjoint subalgebra of $C(X)$. Note that

$$
|f|=\sup \left\{\frac{|f(s)+f(t)|}{2}+\frac{|f(s)-f(t)|}{2} \mid s, t \text { in } X\right\}
$$

defines a norm on $C(X)$, distinct from $\|\cdot\|_{\infty}$, such that $(C(X),|\cdot|)$ is a Banach algebra and $|\cdot|$ satisfies $\frac{1}{4}|f|^{2} \leq\left|f^{2}\right| \leq|f|^{2}$ for all $f$ (since, $\|\cdot\|_{\infty} \leq|\cdot| \leq 2\|\cdot\|_{\infty}$ $[5$, p. 71]). This suggests: Let $\|\cdot\|$ be a norm on $C(X)$ such that (a) $(C(X),\|\cdot\|)$ is a normed linear space (not necessarily complete 'a priori'), and (b) there exist $k_{1}>0, k_{2}>0$ such that $k_{1}\|f\|^{2} \leq\left\|f^{2}\right\| \leq k_{2}\|f\|^{2}(f \in C(X))$. Is $\|\cdot\|$ equivalent to $\|\cdot\|_{\infty}$ ?

(6) A uniform seminorm on an algebra $A$ is a seminorm satisfying all the properties (i)-(iv). Assume $A$ to be a Fréchet commutative locally $m$-convex algebra [7]. Continuity of uniform seminorms on $A$ is tied up with the Michael problem [7, p. 53], viz. is every multiplicative linear functional on $A$ continuous? A simple argument with closed graph theorem shows that there exists a discontinuous multiplicative linear functional on $A$ iff there exists a discontinuous uniform seminorm on $A$. Further, by [9], the topology on $A$ can be assumed to be determined by the collection of all continuous uniform seminorms on $A$. Note that the uniform algebra $\left(C(X),\|\cdot\|_{\infty}\right)$ is known to admit a discontinuous submultiplicative norm [6]. Thus in this version of the Michael problem, 'discontinuous uniform seminorm' cannot be replaced by 'discontinuous submultiplicative seminorm.' On the other hand, a unital commutative topological algebra $B$ may not admit a nonzero multiplicative linear functional, even if $B$ is complete, metrizable, and locally convex [1]. For topological algebra $B$, it is easily seen that $B$ admits a nonzero continuous multiplicative linear functional iff $B$ admits a nonzero continuous uniform seminorm. Further, if $B$ is unital and commutative, this happens iff $B$ admits a nonzero continuous submultiplicative seminorm.

\section{ACKNOWLEDGMENT}

In the first draft of the paper, the main theorem was claimed to hold for any linear associative algebra. There was a gap in the proof. This was pointed out by the referee. The author expresses his sincere thanks to the referee for this contribution, and for several other suggestions. 


\section{REFERENCES}

1. R. Arens, The space $L^{\omega}$ and convex topological rings, Bull. Amer. Math. Soc. 52 (1946), 931-935.

2. B. Aupetit, Properétés spectrales des algèbres de Banach, Lecture Notes in Math. vol. 735, Springer-Verlag, Berlin, Heidelberg, and New York, 1979.

3. S. J. Bhatt and D. J. Karia, Uniqueness of the uniform norm with an application to topological algebras, Proc. Amer. Math Soc. 116 (1992), 499-504.

4. F. F. Bonsall and J. Duncan, Complete normed algebras, Springer-Verlag, Berlin, Heidelberg, and New York, 1973.

5. __ Numerical ranges of operators on normed spaces and of elements of normed algebras, London Math. Soc. Lecture Note Ser., vol. 2, London Math. Soc., Cambridge, 1971.

6. H. G. Dales, $A$ discontinuous homomorphism from $C(X)$, Amer. J. Math. 101 (1979), 647-734.

7. E. Michael, Locally multiplicatively convex topological algebras, Mem. Amer. Math. Soc., vol. 11, Amer. Math. Soc., Providence, RI, 1952.

8. S. Sakai, $C^{*}$-and $W^{*}$-algebras, Springer-Verlag, Berlin, Heidelberg, and New York, 1971.

9. M. Schottenloher, Michael problem and algebras of holomorphic functions, Ark. Math. 37 (1981), 241-247.

10. Z. Sebestyen, Every $C^{*}$-seminorm is automatically submultiplicative, Period. Math Hunger. 10 (1979), 1-8.

Department of Mathematics, Sardar Patel University, Vallabh Vidyanagar - 388120 , GUJARAT, INDIA 